\title{
Impact of the number of aspiration risk factors on mortality and recurrence in community-onset pneumonia
}

\author{
Shingo Noguchi ${ }^{1}$ \\ Kazuhiro Yatera' \\ Tatsuji Kato ${ }^{2}$ \\ Yasuo Chojin² \\ Yoshihisa Fujino ${ }^{3}$ \\ Kentaro Akata' \\ Toshinori Kawanami ${ }^{1}$ \\ Noriho Sakamoto ${ }^{4}$ \\ Hiroshi Mukae ${ }^{4}$ \\ 'Department of Respiratory Medicine, \\ University of Occupational and \\ Environmental Health, Kitakyushu, \\ Japan; ${ }^{2}$ Department of Respiratory \\ Medicine, Tobata Kyoritsu Hospital, \\ Kitakyushu, Japan; ${ }^{3}$ Department of \\ Preventive Medicine and Community \\ Health, School of Medicine, \\ University of Occupational and \\ Environmental Health, Kitakyushu, \\ Japan; ${ }^{4}$ Department of Respiratory \\ Medicine, Unit of Translational \\ Medicine, Nagasaki University \\ Graduate School of Biomedical \\ Sciences, Nagasaki, Japan
}

This article was published in the following Dove Press journal: Clinical Interventions in Aging

Introduction: The clinical significance of the number of aspiration risk factors in patients with pneumonia is unknown as yet. In the present study, we clarify the significance of the number of aspiration risk factors for mortality and recurrence in pneumonia patients.

Methods: This study included 322 patients hospitalized with pneumonia between December 2014 and June 2016. We investigated associations between the number of aspiration risk factors present (orientation disturbance, bedridden, chronic cerebrovascular disease, dementia, sleeping medications and gastroesophageal disease) and 30-day and 6-month mortality, and pneumonia recurrence within 30 days.

Results: Patients were categorized by number of risk factors present into groups of $0-1,2,3$, and 4 or more. Of a total of 322 patients, $93(28.9 \%)$ had $0-1$ risk factors, $112(34.8 \%)$ had 2 , $88(27.3 \%)$ had 3 , and $29(9.0 \%)$ had 4 or more risk factors. The percentages of patients with recurrence of pneumonia were $13.0 \%, 33.0 \%, 43.2 \%$, and $54.2 \%$ in the $0-1,2,3$, and 4 or more risk factor groups, respectively. The percentages of patients with 30-day mortality were $2.2 \%$, $5.4 \%, 11.4 \%$, and $24.1 \%$, and those of patients with 6 -month mortality were $6.6 \%, 24.5 \%$, $30.7 \%$, and $50.0 \%$, in the $0-1,2,3$, and 4 or more risk factor groups, respectively.

Conclusions: The number of aspiration risk factors was associated with increases in both mortality and recurrence in pneumonia patients. Therefore, in clinical practice, physicians should consider not only the presence of aspiration risks but also the number of aspiration risk factors in these patients.

Keywords: aspiration pneumonia, bedridden, dementia, sleeping medications, mortality, recurrence of pneumonia

\section{Introduction}

Aspiration pneumonia is defined as the misdirection of oropharyngeal or gastric content into the lower respiratory tract, ${ }^{1}$ and accounts for $5.0 \%$ to $66.8 \%$ of patients hospitalized with pneumonia. ${ }^{2,3}$ In Japan, the rate of patients diagnosed with aspiration pneumonia tends to be higher than those in other countries, ${ }^{4}$ and aspiration pneumonia is an important problem for an aging population. ${ }^{5}$ However, the mortality rate for patients with aspiration pneumonia has been decreasing in the USA and Spain.,

There have been no sensitive and specific markers for "aspiration risk" or "aspiration pneumonia" in clinical practice so far, ${ }^{8}$ and the definition and gold-standard tests for diagnosing aspiration pneumonia are lacking. ${ }^{1,9}$ Patients with aspiration confirmed by endoscopy and/or video fluoroscopy are reportedly at an 5-7-fold higher risk of pneumonia; ${ }^{10}$ therefore, these methods can be reliable tools for diagnosing aspiration. However, these tools cannot be widely used in elderly patients with pneumonia who are
Correspondence: Shingo Noguchi Department of Respiratory Medicine, University of Occupational and Environmental Health, Japan, I-I, Iseigaoka, Yahatanishiku, Kitakyusyu city, Fukuoka, 807-8555, Japan

Tel +81936917453

Fax +8I 936029373

Emailsn0920@med.uoeh-u.ac.jp 
in poor general condition or without appropriate hospital facilities. Therefore, studies related to aspiration pneumonia have generally been conducted on the basis of patient characteristics that are considered to be aspiration risks and/or radiographic characteristics. ${ }^{1,4,11,12}$ In addition, most of the previous studies related to aspiration pneumonia have investigated the factors associated with aspiration pneumonia, or whether the presence of aspiration pneumonia was associated with mortality. ${ }^{1,2,8,13}$

Two Japanese reports by Maruyama et al and Shindo et al showed that a number of risk factors for multidrugresistant pathogens were associated with 30-day mortality. ${ }^{14,15}$ However, little is known regarding whether the number of aspiration risk factors is useful for estimating short- and long-term mortalities or recurrence of pneumonia.

In the present study, we investigated the significance of the number of aspiration risk factors for mortality and recurrence of pneumonia in patients hospitalized with pneumonia.

\section{Methods}

\section{Study population}

We retrospectively enrolled consecutive patients with community-onset pneumonia who participated in a previously prospective study performed in Tobata Kyoritsu Hospital (218 beds, Kitakyusyu City, Japan) between December 2014 and June 2016 (UMIN 000016179, UMIN 000020371). ${ }^{16}$ Patients whose conditions were complicated with other diseases during hospitalization were excluded. This study was approved by the ethics committee of Tobata Kyoritsu Hospital (No 16-06), and this study did not require written informed consent because it was retrospective in nature and contained no personal information. The age, sex, comorbid diseases, past medical histories, clinical manifestations, and laboratory findings of the participants were collected and recorded.

\section{Definitions of pneumonia}

Pneumonia was diagnosed when the patient met all 3 of the following criteria: 1) at least 1 relevant clinical symptom(s) (fever, cough, sputum production, chest pain); 2) new infiltrates on chest radiograph and/or computed tomography; and 3 ) at least 1 of the designated relevant laboratory findings (white blood cell count $\geq 10,000 / \mu \mathrm{L}$ or increased level of serum C-reactive protein).

\section{Aspiration risk}

In the Japanese Respiratory Society (JRS) Guidelines for the Management of Pneumonia in Adults 2017, ${ }^{17}$ orientation disturbance, bedridden, acute cerebrovascular disease, chronic neurological disorders (dementia, cerebral infarction sequelae, Parkinson disease, etc), iatrogenic causes (sleeping medication, tube feeding, tracheotomy, etc), and gastroesophageal disorders are described as risk factors that predispose aspiration, although there are no gold-standard definitions of aspiration pneumonia. In the present study, the following 6 risk factors were defined as the risk factors for aspiration according to the (JRS) Guidelines: orientation disturbance, European Cooperative Oncology Group-performance status (ECOG-PS) $4^{18}$ (bedridden), chronic cerebrovascular disease including cerebral infarction sequelae and/or Parkinson disease (chronic neurological disorders), dementia (chronic neurological disorders), sleeping medications (iatrogenic causes), and gastroesophageal disease. Acute cerebrovascular disease, tube feeding, and tracheotomy were excluded as aspiration risks because of the small number of such cases. In addition, a patient was defined as having an aspiration risk when the patient had 1 or more of the previously mentioned risk factors in order to evaluate the significance of the presence of aspiration risk.

\section{Outcomes}

In this study, 6-month mortality due to all causes was evaluated as primary outcome, and 30-day mortality on admission and recurrence of pneumonia within 30 days (defined as the emergence of a new episode of pneumonia after remission of the preceding episode ${ }^{16}$ ) were also evaluated. The recurrence of pneumonia within 30 days and 6-month mortality were assessed by reviewing the patients' medical records and/or interviews. The choice of antibiotics was entirely up to the attending physicians.

\section{Statistical analyses}

The STATA 14 software program (StataCorp LP, College Station, TX, USA) was used for the statistical analyses, and Fisher's exact test for tables $(2 \times 2)$ and the Mann-Whitney $U$ test were applied as appropriate. $P<0.05$ was considered statistically significant. The odds ratio (OR) for each outcome for aspiration risk scores of 2, 3, and 4 or more compared with an aspiration risk score of $0-1$ was calculated. In addition, a multivariate analysis was used to identify the most significant variable for the 6 aspiration risk factors. The receiver operating characteristics (ROC) curves and the areas under the curve (AUCs) were analyzed according to the number of aspiration risk factors and each outcome.

\section{Results}

\section{Patient characteristics}

Among 328 hospitalized patients with community-onset pneumonia during the study period, 322 patients were ultimately included in this study (Figure 1). The clinical characteristics of these 322 patients are summarized in 


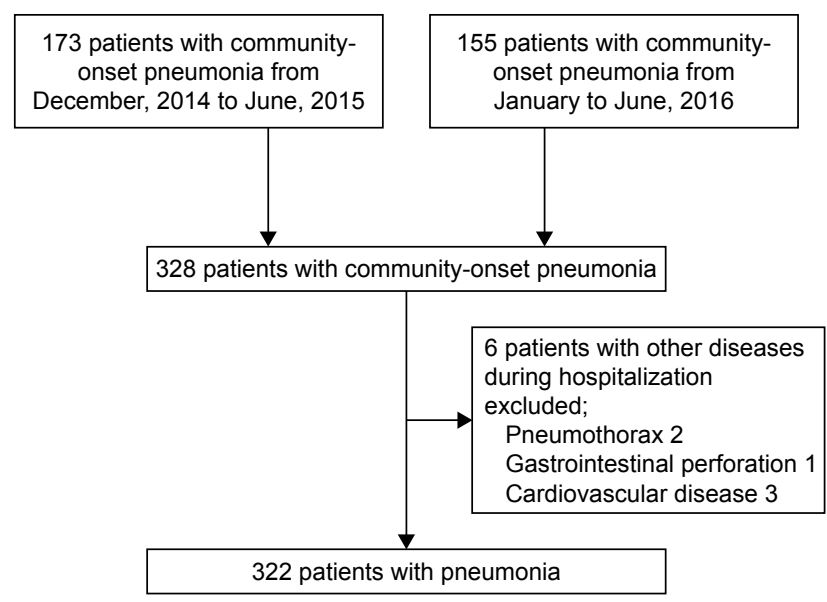

Figure I Entry flow chart.

Table 1. The average age was $83.4 \pm 12.2$ years old, and $57.9 \%$ of patients were female. Among all patients, 286 (88.8\%) were classified as having an aspiration risk (1 or more). The numbers of patients experiencing recurrence of pneumonia within 30 days, 30-day mortality, and 6-month mortality were 95 (31.4\%), 25 (7.8\%), and 63 (23.2\%), respectively.

Table I Characteristics of 322 patients with pneumonia

\begin{tabular}{|c|c|}
\hline Characteristics & Patients with pneumonia \\
\hline Age (years), mean \pm SD & $83.4 \pm 12.2$ \\
\hline Female; n (\%) & $187(58.1)$ \\
\hline ECOG-PS 4; $\mathrm{n}(\%)^{\mathrm{a}}$ & $105(32.6)$ \\
\hline Hospitalization for $\geq 2$ days in the & $80(24.8)$ \\
\hline \multicolumn{2}{|l|}{ preceding 90 days; $\mathrm{n}(\%)$} \\
\hline \multicolumn{2}{|l|}{ Comorbidity; n (\%) } \\
\hline Malignancy & $73(22.7)$ \\
\hline Chronic cerebrovascular disease & $186(57.8)$ \\
\hline Chronic cardiovascular disease & $182(56.5)$ \\
\hline Chronic respiratory disease & $124(38.5)$ \\
\hline Gastroesophageal disease & $57(17.7)$ \\
\hline Chronic liver disease & $18(5.6)$ \\
\hline Chronic kidney disease & $14(4.3)$ \\
\hline Diabetes mellitus & $47(14.6)$ \\
\hline Dementia & $240(74.5)$ \\
\hline Orientation disturbance; n (\%) & II (3.4) \\
\hline Albumin $<3.0 \mathrm{~g} / \mathrm{dL} ; \mathrm{n}(\%)$ & $106(32.9)$ \\
\hline \multicolumn{2}{|l|}{ Radiographic findings; n (\%) } \\
\hline Multilobar involvement $(\geq 2)$ & $107(33.2)$ \\
\hline Pleural effusion & $87(27.0)$ \\
\hline Sleeping medications & $63(19.6)$ \\
\hline Tube feeding & $21(6.5)$ \\
\hline ACE inhibitor drugs & $19(5.9)$ \\
\hline \multicolumn{2}{|l|}{ PSI score; n (\%) } \\
\hline $1-I I I$ & $63(19.6)$ \\
\hline IV & $143(44.4)$ \\
\hline$\vee$ & $116(36.0)$ \\
\hline 30 days mortality & $25(7.8)$ \\
\hline Recurrence of pneumonia within & $95(31.4)$ \\
\hline \multicolumn{2}{|l|}{30 days $^{\mathrm{b}}$} \\
\hline 6-month mortalityc & $63(23.2)$ \\
\hline
\end{tabular}

Notes: ${ }^{\mathrm{E} E C O G-P S 4}$ cannot move at all or perform personal care, all day spent in bed or chair. "Recurrence of pneumonia was evaluated in 303 patients. "6-month mortality was evaluated in 27 I patients.

Abbreviations: ACE, angiotensin-converting enzyme; ECOG-PS, European Cooperative Oncology Group-performance status; PSI, pneumonia severity index.
Numbers of patients in risk factor groups Of the total 322 patients, $93(28.9 \%)$ were in the $0-1$ risk factor group; 112 (34.8\%) had 2 risk factors; 88 (27.3\%) had 3 risk factors; and $29(9.0 \%)$ were in the 4 or more group. Detailed characteristics of patients by number of aspiration risk factors in each category are presented in Table 2.

\section{Recurrence of pneumonia within 30 days, according to the number of aspiration risk factors}

A total of 303 patients were included in the analysis of this outcome because 10 of 322 patients did not admit remission of the first pneumonia and 9 patients lacked the outcome evaluation. The numbers of patients with recurrence of pneumonia within 30 days according to the aspiration risk factor group were 12 of 92 (13.0\%), 35 of $106(33.0 \%), 35$ of $81(43.2 \%)$, and 13 of $24(54.2 \%)$ for the $0-1,2,3$, and 4 or more risk factor groups, respectively (Figure 2A). The ORs in the 2, 3, and 4 or more risk factor groups compared with the $0-1$ risk factor group were 3.29 , 5.07, and 7.88, respectively. An increase in the number of aspiration risk factors was associated with an increase in the rates of pneumonia recurrence within 30 days. In addition, the ORs of univariate and multivariate analyses were similar.

\section{Thirty-day mortality according to the number of aspiration risk factors on admission}

A total of 322 patients were included in the analysis of this outcome. The numbers of patients with 30-day mortality, by risk factor group were 2 of 93 (2.2\%), 6 of $112(5.4 \%)$, 10 of $88(11.4 \%)$, and 7 of $29(24.1 \%)$ for the $0-1,2,3$, and 4 or more risk factor groups, respectively (Figure $2 \mathrm{~B}$ ). The ORs in the 2, 3, and 4 or more risk factor groups compared with the $0-1$ risk factor group were $2.58,5.83$, and 14.5, respectively. An increase in number of aspiration risk factors was associated with an increase in the rates of 30-day mortality. The calculated ORs of univariate and multivariate analyses were similar.

\section{Six-month mortality according to the number of aspiration risk factors}

A total of 271 patients were included in the analysis of this outcome because 25 patients died within 30 days of admission, and 26 lacked the outcome evaluation. The numbers of patients with 6-month mortality according to aspiration risk factor group were 5 of $76(6.6 \%), 24$ of $98(24.5 \%)$, 23 of 75 (30.7\%), and 11 of $22(50.0 \%)$, for the $0-1,2,3$, 
Table 2 The number of aspiration risk factors in each category

\begin{tabular}{|c|c|c|c|c|}
\hline Characteristics & $\begin{array}{l}\text { Risk 0-I } \\
(n=93)\end{array}$ & $\begin{array}{l}\text { Risk } 2 \\
(n=|| 2)\end{array}$ & $\begin{array}{l}\text { Risk } 3 \\
(\mathrm{n}=88)\end{array}$ & $\begin{array}{l}\text { Risk } \geq 4 \\
(n=29)\end{array}$ \\
\hline Age (years), mean $\pm \mathrm{SD}$ & $75.8 \pm 16.7$ & $86.6 \pm 8.6$ & $86.7 \pm 7.7$ & $85.8 \pm 6.9$ \\
\hline Female; n (\%) & $48(51.6)$ & $67(59.8)$ & $56(63.6)$ & $16(55.2)$ \\
\hline $\begin{array}{l}\text { Hospitalization for } \geq 2 \text { days in the } \\
\text { preceding } 90 \text { days; } n(\%)\end{array}$ & $16(17.2)$ & $30(26.8)$ & $23(26.1)$ & II (37.9) \\
\hline \multicolumn{5}{|l|}{ Comorbidity; n (\%) } \\
\hline Malignancy & $19(20.4)$ & $23(20.5)$ & $23(26.1)$ & $8(27.6)$ \\
\hline Chronic cardiovascular disease & $40(43.0)$ & $68(60.7)$ & $58(65.9)$ & $16(55.2)$ \\
\hline Chronic respiratory disease & $35(37.6)$ & $50(44.6)$ & $26(29.5)$ & $13(44.8)$ \\
\hline Chronic liver disease & $5(5.4)$ & $4(3.6)$ & $7(8.0)$ & $2(6.9)$ \\
\hline Chronic kidney disease & $\mathrm{I}(\mathrm{I} . \mathrm{I})$ & $5(4.5)$ & $4(4.5)$ & $4(13.8)$ \\
\hline Diabetes mellitus & $14(15.1)$ & $14(12.5)$ & $10(11.4)$ & $9(31.0)$ \\
\hline Albumin $<3.0 \mathrm{~g} / \mathrm{dL}$ & $19(20.4)$ & $35(31.3)$ & $38(43.2)$ & $14(48.3)$ \\
\hline \multicolumn{5}{|l|}{ Radiographic findings; $\mathrm{n}(\%)$} \\
\hline Multilobar involvement $(\geq 2)$ & $32(34.4)$ & $36(32.1)$ & $30(34.1)$ & $9(31.0)$ \\
\hline Pleural effusion & $16(17.2)$ & $28(25.0)$ & $31(35.2)$ & $12(41.4)$ \\
\hline Tracheotomy & $0(0.0)$ & $0(0.0)$ & $\mathrm{I}(\mathrm{I} . \mathrm{I})$ & I (3.4) \\
\hline Tube feeding & $2(2.2)$ & $4(3.6)$ & $10(11.4)$ & $5(17.2)$ \\
\hline ACE inhibitor drugs & $3(3.2)$ & $6(5.4)$ & $6(6.8)$ & $4(13.8)$ \\
\hline \multicolumn{5}{|l|}{ PSI score; n (\%) } \\
\hline I-III & $42(45.2)$ & $15(13.4)$ & $5(5.7)$ & $\mathrm{I}(3.4)$ \\
\hline IV & $34(36.6)$ & $55(49.1)$ & $43(48.9)$ & II (37.9) \\
\hline $\mathrm{V}$ & $17(18.3)$ & $42(37.5)$ & $40(45.5)$ & $17(58.6)$ \\
\hline \multicolumn{5}{|c|}{ Aspiration risk factors in this study; $\mathrm{n}(\%)$} \\
\hline Orientation disturbance & $2(2.2)$ & $5(4.5)$ & $\mathrm{I}(\mathrm{I} . \mathrm{I})$ & $3(10.3)$ \\
\hline ECOG-PS4 ${ }^{\mathrm{a}}$ & $\mathrm{I}(\mathrm{I} .1)$ & $19(17.0)$ & $58(65.9)$ & $27(93.1)$ \\
\hline Chronic cerebrovascular disease & $12(12.9)$ & $68(60.7)$ & $78(88.6)$ & $28(96.6)$ \\
\hline Dementia & $28(30.1)$ & $98(87.5)$ & $85(96.6)$ & $29(100.0)$ \\
\hline Sleeping medications & $5(5.4)$ & 20 (I7.9) & $23(26.1)$ & $15(51.7)$ \\
\hline Gastroesophageal disease & $9(9.7)$ & $14(12.5)$ & $19(21.6)$ & $15(51.7)$ \\
\hline
\end{tabular}

Note: ${ }^{a}$ ECOG-PS4 cannot move at all or perform personal care, all day spent in bed or chair.

Abbreviations: ACE, angiotensin-converting enzyme; ECOG-PS, European Cooperative Oncology Group-performance status; PSI, pneumonia severity index.

and 4 or more risk factor groups, respectively (Figure 2C). The ORs in the 2, 3, and 4 or more risk factor groups compared with the 0-1 risk factor group were 4.61, 6.28, and 14.2, respectively. An increase in the number of the aspiration risk factors was associated with an increase in the rates of 6-month mortality. The OR results of univariate and multivariate analyses were similar. On the other hand, the 6-month mortality in patients with recurrence of pneumonia (50.6\%) was significantly higher $(P<0.0001)$ than in patients without recurrence of pneumonia $(9.8 \%)$.
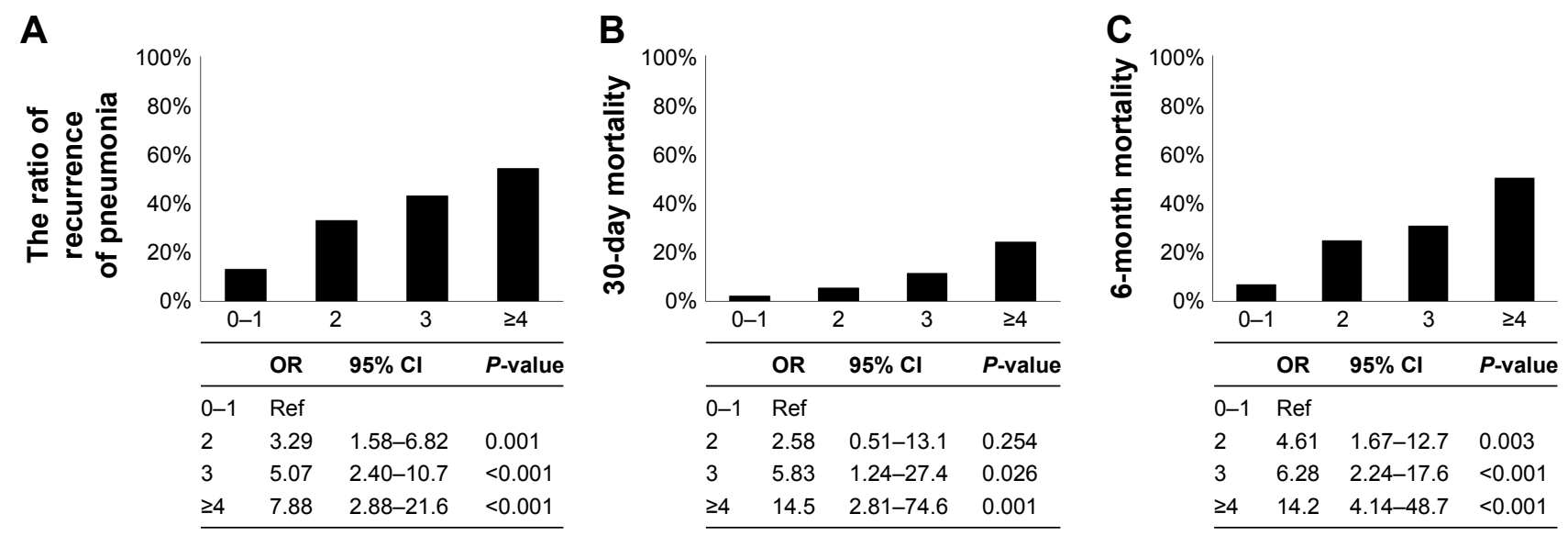

Figure 2 The ratio of each outcome and odds ratio according to the number of aspiration risk factors. Note: (A) Recurrence of pneumonia within 30 days, (B) 30-day mortality, (C) 6-month mortality. Abbreviations: OR, odds ratio; Ref, reference. 


\section{Prognostic accuracy for each outcome according to the number of aspiration risk factors}

The relationship between the rates of mortality due to pneumonia or recurrence, and the presence of aspiration risk factors is shown in Figure 3A, C, and E. In addition, the ROC curve for each outcome by the number of aspiration risk factors is shown in Figure 3B, D, and F. There were significant differences in the recurrence of pneumonia within 30 days and the 6-month mortality between patients with 1 or more aspiration risk factors and those without any such factors $(0)$. However, no significant differences were found in the 30-day mortality between these 2 groups. The AUCs using ROC curves for the recurrence of pneumonia within 30 days and the 30-day and 6-month mortalities were 0.67 (95\% CI, 0.61-0.73), 0.72 (95\% CI, 0.61-0.82), and 0.68 (95\% CI, 0.62-0.75), respectively.
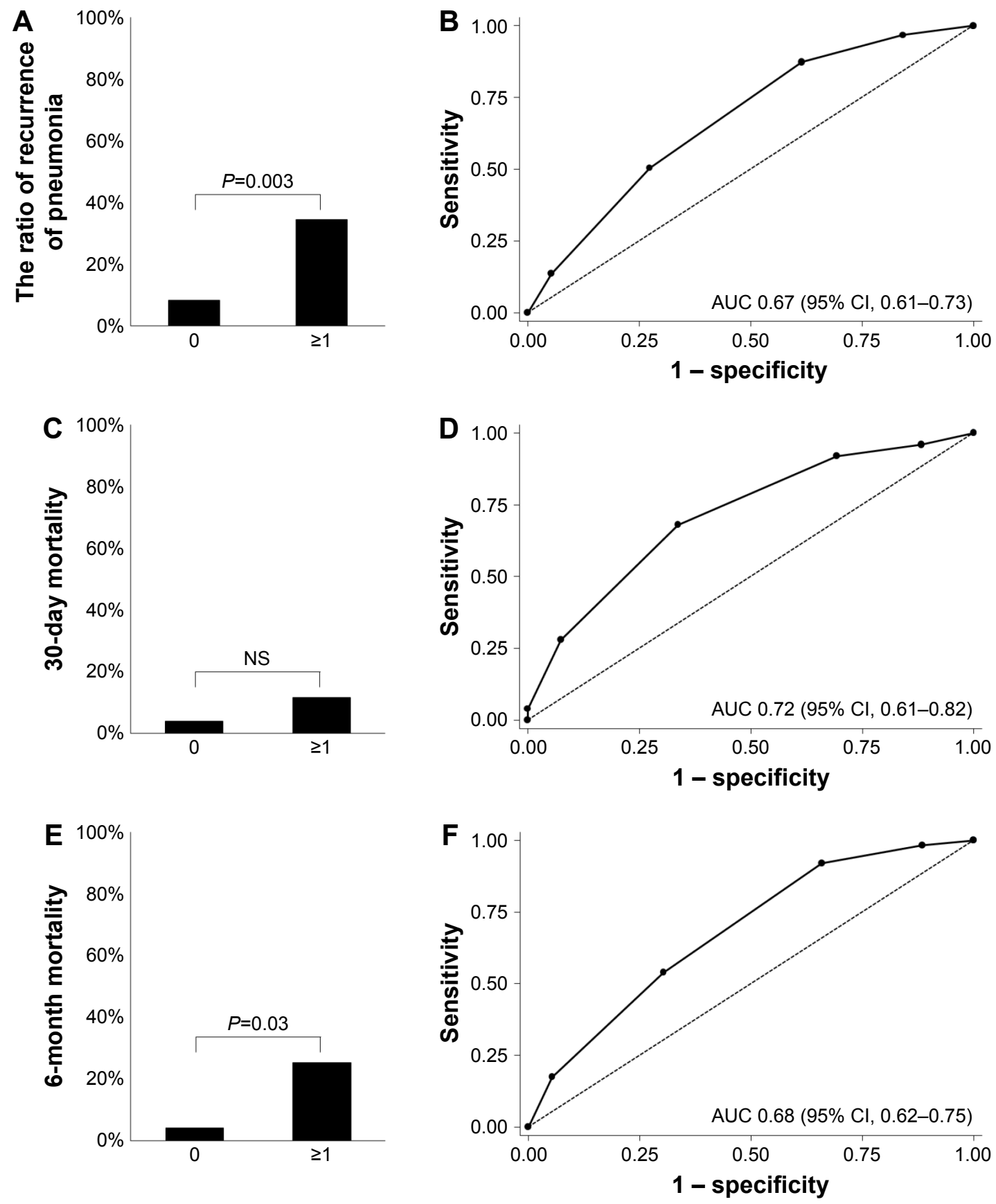

Figure 3 The ratio for each outcome according to the aspiration risk.

Notes: (A) Recurrence of pneumonia within 30 days. (B) The receiver operating characteristics for recurrence of pneumonia. (C) 30 -day mortality. (D) The receiver operating characteristics for 30-day mortality. (E) 6-month mortality. (F) The receiver operating characteristics for 6-month mortality.

Abbreviations: AUC, areas under the curve; NS, not significant. 


\section{Multivariate analysis for 6 aspiration risk factors}

We evaluated whether the 6 aspiration risk factors we used in this study (orientation disturbance, bedridden on ECOG-PS 4, chronic cerebrovascular disease, dementia, sleeping medications, and gastroesophageal disease) are independent risk factors for pneumonia patients with aspiration risk. In this analysis, we defined patients with "abnormal swallowing ability" as pneumonia patients with aspiration risk using the modified water test, ${ }^{19}$ which was performed before discharge. We evaluated the 6 aspiration risk factors using multivariate analysis for the 268 of 322 patients in whom the modified water test was performed. As a result, ECOG-PS 4 (OR: 3.31; 95\% CI: 1.83-6.00, $P<0.001$ ), dementia (OR: 5.26, 95\% CI: 2.30-12.03, $P<0.001$ ), and sleeping medications (OR: $2.08,95 \%$ CI: $1.04-4.14$, $P=0.038$ ) were significant independent risk factors for pneumonia patients with aspiration risk.

\section{Discussion}

The present study investigated the clinical significance of the number of aspiration risk factors for mortality and the recurrence of pneumonia in clinical practice for patients with community-onset pneumonia. The results of this study indicated that the number of aspiration risk factors is associated with the rate of short-term (30 days) and long-term (6 months) mortalities, as well as the rate of pneumonia recurrence within 30 days.

Aspiration pneumonia generally occurs as a result of multifocal risk factors, ${ }^{4}$ and various studies have reported aspiration risk factors and their relation to the mortality and/or recurrence of pneumonia. ${ }^{1,2,8,13}$ However, there have been only a few reports evaluating the relationship between mortality and the recurrence of pneumonia, and the number of aspiration risk factors. In addition, the clinical significance of the number of aspiration risk factors in patients with pneumonia is unknown as yet. We clarified that the number of aspiration risk factors we used was related to an increase in the rate of short- and long-term mortalities, and the recurrence of pneumonia in the present study. A recent report by Kawai et al showed a significant relationship between an increase in the number of aspiration risks and an increase in the incidence of pneumonia among patients who had received treatment for head and neck cancer. ${ }^{20}$ In addition, Weiss et al reported that an increase in the amylase level of bronchoalveolar lavage fluid, which is 1 marker for aspiration, was significantly related to increased number of aspiration risk factors (swallowing dysfunction, altered consciousness, cardiac arrest, difficult intubation, and periintubation vomiting). ${ }^{21}$ Aspiration risk factors are crossassociated among themselves and exhibit collinearity with each other, ${ }^{4}$ and the results of our study may demonstrate a positive association between the combination of various aspiration risk factors and an increase in mortality and recurrence of pneumonia in patients with community-onset pneumonia.

Studies related to aspiration pneumonia have often been conducted on the basis of patient characteristics that are thought to be aspiration risks and/or radiographic characteristics. ${ }^{1,4,11,12}$ The definitive criteria recognized for aspiration pneumonia are still lacking,,$^{1,9}$ although the 6 aspiration risks used in this study were based on the pneumonia guidelines published by the JRS recently. Therefore, we verified the adequacy of these 6 aspiration risks using the results of a modified water test before discharge. Among the 6 risk factors we used in this study, ECOG-PS 4 (OR: 3.31; $P<0.001$ ), dementia (OR: 5.26; $P<0.001$ ), and sleeping medications (OR: $2.08 ; P=0.038)$ were identified as independent risk factors in multivariate analysis (Table 3 ). Conversely, orientation disturbance and comorbidity (chronic cerebrovascular disease and gastroesophageal disease) did not demonstrate significant differences for aspiration risk in this study. Similar to our results, Manabe et al reported in a study with a larger number of elderly patients that comorbid diseases did not affect the incidence of aspiration pneumonia, and that activity of daily living might be important for aspiration pneumonia. ${ }^{13}$ These results might also be influenced by the higher percentage of advanced-age patients in the study. On the contrary, it is widely known that confusion and comorbidities such as cerebrovascular diseases and gastroesophageal diseases are associated with aspiration pneumonia. ${ }^{8,22} \mathrm{We}$ also think these are important factors for aspiration, although there were no significant differences for these factors in this study. Therefore, we believe that

Table 3 The evaluation of 6 risk factors using multivariate analysis

\begin{tabular}{llll}
\hline Variables & \multicolumn{3}{l}{ Multivariate analysis } \\
\cline { 2 - 4 } & Odds ratio & $\mathbf{9 5 \%} \mathbf{C l}$ & $\mathbf{P}$-value \\
\hline Orientation disturbance & 0.52 & $0.10-2.62$ & 0.425 \\
Chronic cerebrovascular disease & 1.51 & $0.86-2.68$ & 0.153 \\
Dementia & 5.26 & $2.30-12.03$ & $<0.00 \mathrm{I}$ \\
ECOG-PS 4 & 3.31 & $1.83-6.00$ & $<0.00 \mathrm{I}$ \\
Sleeping medications & 2.08 & $1.04-4.14$ & 0.038 \\
Gastroesophageal disease & 1.15 & $0.56-2.35$ & 0.709 \\
\hline
\end{tabular}

Abbreviation: ECOG-PS, European Cooperative Oncology Group-performance status. 
the 6 factors we used in this study can be essential factors for studies of aspiration pneumonia. Further investigations are necessary to elucidate adequate or better aspiration risk factors for evaluating several risks in patients with aspiration pneumonia.

The presence of aspiration risk factors showed significant differences in 6-month mortality and 30-day recurrence of pneumonia; in addition, the 6-month mortality in patients with 30-day recurrence of pneumonia was significantly higher than that in patients without 30-day recurrence of pneumonia. The Adult Pneumonia Study Group in Japan also showed that the presence of pneumonia recurrence was associated with a poor prognosis. ${ }^{23} \mathrm{We}$, therefore, believe that the existence of any aspiration risk factors is itself an important factor influencing the long-term prognosis in elderly patients with pneumonia. Whereas, the presence of risk factors was not significantly associated with the 30-day mortality in our study. A recent systematic review of aspiration risk factors by Komiya et al showed that aspiration pneumonia was related to short- (not requiring the intensive care unit) and long-term mortality, and recurrent pneumonia, ${ }^{24}$ but they also demonstrated that the results in each study varied widely with respect to shortterm mortality, which was strongly influenced by differences in study size, population, and the definition of aspiration pneumonia used; ${ }^{24}$ our results for short-term mortality were also influenced by these factors. Therefore, selection and use of better aspiration risk factors for predicting mortality and recurrence of pneumonia might lead to better prognosis in pneumonia patients with aspiration risks, and further studies are expected.

There are several limitations associated with the present study. First, no unified definition of aspiration pneumonia has yet been established. However, aspiration risks used in this study were considered to be established based on the JRS guidelines; therefore, we believe that our findings are significant and reliable. Second, this study was a single-center trial, although the number of enrolled patients was relatively large.

In conclusion, our results may indicate that the number of aspiration risk factors is associated with an increase in mortality and the recurrence of pneumonia, especially among elderly patients. Consideration of not only the presence of aspiration risks, but also the number of aspiration risk factors, is important for better clinical practice in patients with pneumonia. Further prospective multicenter studies are necessary to confirm our results. In addition, better aspiration risk factors are expected to provide increased understanding, and strategic and effective care for pneumonia patients with aspiration risks.

\section{Disclosure}

The authors report no conflicts of interest in this work.

\section{References}

1. Taylor JK, Fleming GB, Singanayagam A, Hill AT, Chalmers JD. Risk factors for aspiration in community-acquired pneumonia: analysis of a hospitalized UK cohort. Am J Med. 2013;126(11):995-1001.

2. Hayashi M, Iwasaki T, Yamazaki Y, et al. Clinical features and outcomes of aspiration pneumonia compared with non-aspiration pneumonia: a retrospective cohort study. J Infect Chemother. 2014;20(7):436-442.

3. DiBardino DM, Wunderink RG. Aspiration pneumonia: a review of modern trends. J Crit Care. 2015;30(1):40-48.

4. Komiya K, Ishii H, Kadota J. Healthcare-associated Pneumonia and Aspiration Pneumonia. Aging Dis. 2014;6(1):27-37.

5. Morimoto K, Suzuki M, Ishifuji T, et al. The burden and etiology of community-onset pneumonia in the aging Japanese population a multicenter prospective study. PLoS One. 2015;10(3):e0122247.

6. Wu CP, Chen YW, Wang MJ, Pinelis E. National trends in admission for aspiration pneumonia in the United States in 2002-2012. Ann Am Thorac Soc. 2017;14(6):874-879.

7. Palacios-Cena D, Hernandez-Barrera V, Lopez-de-Andres A, et al. Time trends in incidence and outcomes of hospitalizations for aspiration pneumonia among elderly people in Spain (2003-2013). Eur J Intern Med. 2017;38:61-67.

8. Lanspa MJ, Peyrani P, Wiemken T, Wilson EL, Ramirez JA, Dean NC. Characteristics associated with clinician diagnosis of aspiration pneumonia: a descriptive study of afflicted patients and their outcomes. J Hosp Med. 2015;10(2):90-96.

9. Bosch X, Formiga F, Cuerpo S, Torres B, Roson B, Lopez-Soto A. Aspiration pneumonia in old patients with dementia. Prognostic factors of mortality. Eur J Intern Med. 2012;23(8):720-726.

10. Schmidt J, Holas M, Halvorson K, Reding M. Videofluoroscopic evidence of aspiration predicts pneumonia and death but not dehydration following stroke. Dysphagia. 1994;9(1):7-11.

11. Hayashi M, Iwasaki T, Yamazaki Y, et al. Clinical features and outcomes of aspiration pneumonia compared with non-aspiration pneumonia: a retrospective cohort study. J Infect Chemother. 2014;20(7):436-442.

12. Cabre M, Serra-Prat M, Palomera E, Almirall J, Pallares R, Clave P. Prevalence and prognostic implications of dysphagia in elderly patients with pneumonia. Age Ageing. 2010;39(1):39-45.

13. Manabe T, Teramoto S, Tamiya N, Okochi J, Hizawa N. Risk factors for aspiration pneumonia in older adults. PLoS One. 2015;10(10):e0140060.

14. Shindo Y, Ito R, Kobayashi D, et al. Risk factors for drug-resistant pathogens in community-acquired and healthcare-associated pneumonia. Am J Respir Crit Care Med. 2013;188(8):985-995.

15. Maruyama T, Fujisawa T, Okuno M, et al. A new strategy for healthcareassociated pneumonia: a 2-year prospective multicenter cohort study using risk factors for multidrug-resistant pathogens to select initial empiric therapy. Clin Infect Dis. 2013;57(10):1373-1383.

16. Chojin Y, Kato T, Rikihisa M, et al. Evaluation of the mann assessment of swallowing ability in elderly patients with pneumonia. Aging Dis. 2017;8(4):420-433.

17. The Japanese Respiratory Society. The JRS Guidelines for the Management of Pneumonia in Adults 2017. Available from: http://www. jrs.or.jp/modules/guidelines/index.php?content_id=94. Accessed November 21, 2017.

18. Oken MM, Creech RH, Tormey DC, et al. Toxicity and response criteria of the Eastern Cooperative Oncology Group. Am J Clin Oncol. 1982; 5(6):649-655.

19. Tohara H, Saitoh E, Mays KA, Kuhlemeier K, Palmer JB. Three tests for predicting aspiration without videofluorography. Dysphagia. 2003; 18(2):126-134.

20. Kawai S, Yokota T, Onozawa Y, et al. Risk factors for aspiration pneumonia after definitive chemoradiotherapy or bio-radiotherapy for locally advanced head and neck cancer: a monocentric case control study. BMC Cancer. 2017;17(1):59. 
21. Weiss CH, Moazed F, DiBardino D, Swaroop M, Wunderink RG. Bronchoalveolar lavage amylase is associated with risk factors for aspiration and predicts bacterial pneumonia. Crit Care Med. 2013;41(3): 765-773.

22. Marik PE, Kaplan D. Aspiration pneumonia and dysphagia in the elderly. Chest. 2003;124(1):328-336.
23. Ishifuji T, Sando E, Kaneko N, et al; Adult Pneumonia Study Group Japan (APSG-J). Recurrent pneumonia among Japanese adults: disease burden and risk factors. BMC Pulm Med. 2017;17(1):12.

24. Komiya K, Rubin BK, Kadota JI, et al. Prognostic implications of aspiration pneumonia in patients with community acquired pneumonia: a systematic review with meta-analysis. Sci Rep. 2016;6:38097.

\section{Publish your work in this journal}

Clinical Interventions in Aging is an international, peer-reviewed journal focusing on evidence-based reports on the value or lack thereof of treatments intended to prevent or delay the onset of maladaptive correlates of aging in human beings. This journal is indexed on PubMed Central, MedLine,

\section{Dovepress}

CAS, Scopus and the Elsevier Bibliographic databases. The manuscript management system is completely online and includes a very quick and fair peer-review system, which is all easy to use. Visit http://www.dovepress. com/testimonials.php to read real quotes from published authors. 